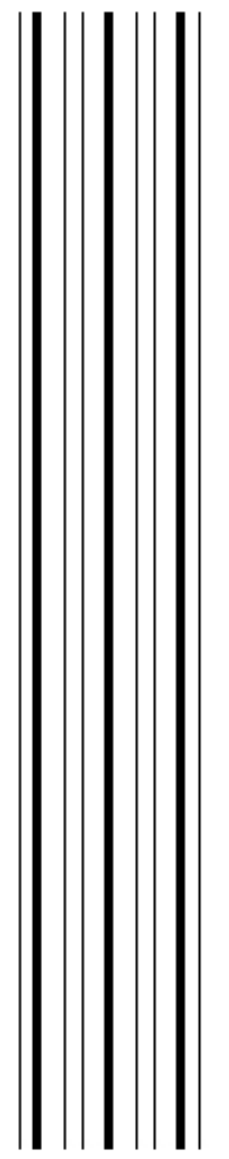

\title{
POINT 2012: ENDF/B-VII.1 Final Temperature Dependent Cross Section Library
}

\section{A Brief Library Summary}

\author{
by \\ Dermott E. Cullen \\ Lawrence Livermore National Laboratory \\ P.O.Box 808/L-198 \\ Livermore, CA 94550
}

January 7, 2012

U.S. Department of Energy

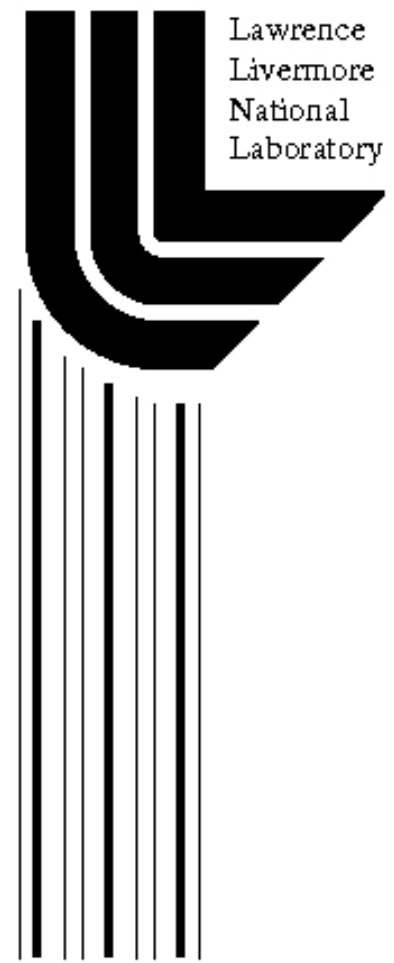




\section{DISCLAIMER}

This document was prepared as an account of work sponsored by an agency of the United States government. Neither the United States government nor Lawrence Livermore National Security, LLC, nor any of their employees makes any warranty, expressed or implied, or assumes any legal liability or responsibility for the accuracy, completeness, or usefulness of any information, apparatus, product, or process disclosed, or represents that its use would not infringe privately owned rights. Reference herein to any specific commercial product, process, or service by trade name, trademark, manufacturer, or otherwise does not necessarily constitute or imply its endorsement, recommendation, or favoring by the United States government or Lawrence Livermore National Security, LLC. The views and opinions of authors expressed herein do not necessarily state or reflect those of the United States government or Lawrence Livermore National Security, LLC, and shall not be used for advertising or product endorsement purposes.

This work performed under the auspices of the U.S. Department of Energy by Lawrence Livermore National Laboratory under Contract DE-AC52-07NA27344. 


\title{
POINT 2012: ENDF/B-VII.1 Final Temperature Dependent Cross Section Library
}

\section{A Brief Library Summary}

\author{
by \\ Dermott E. Cullen \\ Lawrence Livermore National Laboratory \\ P.O.Box 808/L-198 \\ Livermore, CA 94550
}

January 7, 2012

Table of Contents

1) A Brief Library Summary

2) Overview

3) Introduction: POINT 2012: ENDF/B-VII.1 Final

4) PREPRO 2012 Codes

5) Data Processing

6) Accuracy of Results

7) Contents of the Library

8) Requesting POINT 2012 Data

9) Installation and Use of POINT 2012

10) Acknowledgments

11) References

12) Appendix A: Contents of ENDF/B-VII.1

13) Appendix B: Elemental vs. Isotopic Evaluations

14) Appendix C: Completeness of VII.1 and VII.0

15) Appendix D: Summary of $\langle v(E)>$ for all isotopes in ENDF/B-VII.1 and VII.0

16) Appendix E: The Effects of Temperature and Doppler Broadening 


\section{A Brief Library Summary}

This is merely an informal memo to briefly summarize the contents of the new ENDF/B-VII.1 Final library, released in December 2011; focus here is only on the quantity of data. A later document to planned to more completely document the quality of this new library.

\section{Overview}

This report is one in the series of "POINT" reports that over the years have presented temperature dependent cross sections for the then current version of ENDF/B [R1]. In each case I have used my personal computer at home and publicly available data and codes

1) publicly available nuclear data (the current ENDF/B data, available on-line at the National Nuclear Data Center, Brookhaven National Laboratory, http://www.nndc.bnl.gov/) and,

2) publicly available computer codes (the current PREPRO codes, available on-line at the Nuclear Data Section, IAEA, Vienna, Austria,

http://www-nds.iaea.or.at/ndspub/endf/prepro/) and,

3) My own personal computer located in my home.

I have used these in combination to produce the temperature dependent cross sections used in applications and described in this report. I should mention that today anyone with a personal computer can produce these results: by its very nature I consider this data to be born in the public domain.

\section{Introduction: POINT 2012: ENDF/B-VII.1 Final}

The latest ENDF/B-VII.1 Final data library was recently released and is now freely available through the National Nuclear Data Center (NNDC), Brookhaven National Laboratory. This release completely supersedes all preceding releases of ENDF/B.

Individual files and/or the complete tarball can also be downloaded from:

http://www.nndc.bnl.gov/

Compared to VII.0 which included 393 evaluations, VII.1 included 423 evaluations; this includes 32 new evaluations; 2 natural evaluations were dropped (23-V - Nat, and 30-Zn-Nat). The 32 new evaluations are; note the isotopes of $23-\mathrm{V}$ and $30-\mathrm{Zn}$; these have replaced the natural evaluations in VII.0.

32 New Evaluation in VII.1 (not in VII.0)

\begin{tabular}{|l|l|l|l|l|l|l|l|}
\hline $23-V-50$ & $30-Z n-66$ & $69-T m-168$ & $74-W-180$ & $91-P a-229$ & $93-N p-234$ & $97-B k-246$ \\
$23-V-51$ & $30-Z n-67$ & $69-T m-169$ & $81-T l-203$ & $91-P a-230$ & $95-A m-240$ & $97-B k-247$ \\
$30-Z n-64$ & $30-Z n-68$ & $69-T m-170$ & $81-T l-205$ & $92-U-230$ & $96-C m-240$ & $97-B k-248$ \\
$30-Z n-65$ & $30-Z n-70$ & $73-T a-180$ & $90-T h-231$ & $92-U-231$ & $97-B k-245$ & $99-$-Es-251 \\
& & & & & & $98-C f-246$ & $99-E s-254 m$ \\
\hline
\end{tabular}


423 Evaluations in VII.1 (32 new evaluations in RED)

\begin{tabular}{|c|c|c|c|c|c|c|c|c|c|}
\hline $1-H-1$ & 20-Ca-44 & 32-Ge-74 & 42-Мо-92 & 49-In-115 & 54-Хе-131 & 61-Pm-147 & 68-Er-166 & 88-Ra-224 & 94-Pu-242 \\
\hline 1-H -2 & 20-Ca-46 & 32-Ge-76 & 42-Mo-94 & $50-S n-112$ & 54-Хe-132 & 61-Pm-148 & 68-Er-167 & 88-Ra-225 & 94-Pu-243 \\
\hline 1-H -3 & 20-Ca-48 & 33-As-74 & 42-Mo-95 & & J4-Ае-1J2 & OT-16I-140 & 68-Er-168 & 88-Ra-226 & 94-Pu-244 \\
\hline 2-He-3 & $21-S c-45$ & 33-As-75 & 42-Mo-96 & $50-\mathrm{Sn}-114$ & -153 & m-148m & 68-Er-170 & 89-Ac-225 & 94-Pu-246 \\
\hline $2-\mathrm{He}-4$ & 22-Ti-46 & 34-Se-74 & 42-Mo-97 & $\begin{array}{l}50-S n-114 \\
50-S n-115\end{array}$ & 54-Xe-134 & 61-Pm & 69-Tm-168 & 89-Ac-226 & 95-Am-240 \\
\hline 3-Li-6 & 22-Ti-47 & 34-Se-76 & 42-M0-98 & $\begin{array}{l}50-S n-115 \\
50-4 n-116\end{array}$ & 54-Xe-135 & 61-Pm-151 & 69-Tm-169 & 89-Ac-227 & 95-Am-241 \\
\hline 3-Li-7 & 22-Ti-48 & $\begin{array}{l}\text { 34-Se-10 } \\
\text { 34-Se-77 }\end{array}$ & $\begin{array}{l}42-110-90 \\
42-10-99\end{array}$ & 50-Sn-116 & $54-X e-136$ & 62-Sm-144 & 69-Tm-170 & 90-Th-227 & 95-Am-242 \\
\hline 4-Be-7 & 22-Ti-49 & $34-S e-78$ & 42-110-99 & 50-Sn-117 & $55-C s-133$ & 62-Sm-147 & 71-Lu-175 & 90-Th-228 & 95-Am-242m \\
\hline 4-Ве-9 & 22-Ti-50 & $\begin{array}{l}34-\mathrm{se}-10 \\
0\end{array}$ & 42-Mo-100 & 50-Sn-118 & Cs-134 & 62-Sm-148 & 71-Lu-176 & 90-Th-229 & 95-Am-243 \\
\hline 5-B - 10 & $\begin{array}{l}22-11-50 \\
23-V-50\end{array}$ & 34-Se-79 & 43-Тc-99 & 50-Sn-119 & 55-Cs-135 & 62-Sm-149 & 72 & 90-Th-230 & 95-Am-244 \\
\hline $5-8-11$ & $23-V-50$ & 34-Se-80 & 44-Ru-96 & 50-Sn-120 & 55-Cs-136 & 62-Sm-150 & 7 & $90-$ Th-231 & 95-Am-244n \\
\hline 5-B -11 & $23-V-51$ & 34-Se-82 & 44-Ru-98 & 50-Sn-122 & 55-Cs-137 & 62-Sm-151 & & 90-Th-232 & 96-Cm-24 \\
\hline 6-C -Nat & 24-Cr-50 & 35-Br-79 & 44-Ru-99 & $50-S n-123$ & 56-Ba-130 & 62-Sm-152 & & & $90-\mathrm{cm}-2$ \\
\hline $7-N-14$ & 24-Cr-52 & 35-Br-81 & 44-Ru-100 & 50-Sn-124 & 56-Ba-132 & 62-Sm-153 & 78 & 3 & 96-Cn \\
\hline $7-\mathrm{N}-15$ & 24-Cr-53 & $36-\mathrm{Kr}-78$ & 44-Ru-101 & 50-Sn-125 & 56-Ba-133 & 62-Sm-154 & 79 & 90-Th-234 & 96-Cm-242 \\
\hline $8-0-16$ & 24-Cr-54 & $36-\mathrm{Kr}-80$ & 44-Ru-102 & 50-Sn-126 & 56-Ba-134 & 63-Eu-151 & 72-Hf-180 & 91-Pa-229 & 96-Cm-243 \\
\hline 8-O -17 & 25-Mn-55 & $36-\mathrm{Kr}-82$ & 44-Ru-103 & 51-Sb-121 & 56-Ba-135 & 63-Eu-152 & 73-Та-180 & 91-Pa-230 & 96-Cm-244 \\
\hline 9-F -19 & 26-Fe-54 & $36-K r-83$ & 44-Ru-104 & $51-S b-123$ & 56-Ba-136 & 63-F & 73-Тa-181 & 91-Pa-231 & 96-Cm-245 \\
\hline 11-Na-22 & $26-\mathrm{Fe}-56$ & $36-\mathrm{Kr}-84$ & 44-Ru-105 & $51-S b-124$ & 5 & 00 & 73-Тa-182 & 91-Pa-232 & 96-Cm-246 \\
\hline 11-Na-23 & 26-Fe-57 & 36-Kr-85 & 44-Ru-106 & $\begin{array}{l}51-S D-124 \\
51-S b-125\end{array}$ & 56-15a-137 & 63 & 74-W -180 & 91-Pa-233 & 96-Cm-247 \\
\hline 12-Mg-24 & $26-\mathrm{Fe}-58$ & $36-K r-86$ & $\begin{array}{l}44-K u-100 \\
45-R h-103\end{array}$ & $\begin{array}{l}51-5 b-125 \\
51-5 b 126\end{array}$ & 56-Ba-138 & 63-Eu-155 & $74-W-182$ & $92-U-230$ & 96-Cm-248 \\
\hline & & 50-KY- & 45-Rh-103 & 51-Sb-126 & 56-Ba-140 & 63-Eu-156 & $74-W-183$ & $92-U-231$ & 96-Cm-24 \\
\hline 12-Mg-25 & 27-Co-58 & 37-Rb-85 & 45-Rh-105 & 52-Te-120 & 57-La-138 & 63-Eu-157 & $74-W-184$ & $92-U-232$ & $96-\mathrm{Cm}-2$ \\
\hline 12-Mg-26 & 27-Co-58m & 37-Rb-86 & 46-Pd-102 & 52-Тe-122 & 57-La-139 & 64-Gd-152 & 104 & 433 & 90-Cm- \\
\hline 13-Al-27 & 27-Co-59 & 37-Rb-87 & 46-Pd-104 & 52-Te-123 & 57-La-140 & 64-Gd-153 & & 53 & \\
\hline 14-Si-28 & 28-Ni-58 & 38-Sr-84 & 46-Pd-105 & 52-Te-124 & 58-Ce-136 & 64-Gd-154 & 85 & -234 & 97-Bk-246 \\
\hline 14-Si-29 & 28-Ni-59 & 38-Sr-86 & 46-Pd-106 & 52-Te-125 & 58-Ce-138 & 64-Gd-155 & 75-Re-187 & 92-U -235 & 97-Bk-247 \\
\hline 14-Si-30 & 28-Ni-60 & 38-Sr-87 & 46-Pd-107 & 52-Te-126 & 58-Ce-139 & 64-Gd-156 & 77-Ir-191 & 92-U -236 & 97-Bk-248 \\
\hline 15-P -31 & 28-Ni-61 & 38-Sr-88 & 46-Pd-108 & 52-Te-127m & 58-Ce-140 & 64-Gd-157 & 77-Ir-193 & 92-U -237 & 97-Bk-249 \\
\hline $16-S-32$ & $28-\mathrm{Ni}-62$ & 38-Sr-89 & 46-Pd-110 & 52-Тe-128 & 58-Ce-141 & 64-Gd. & 79-Au-197 & $92-U-238$ & 97-Bk-250 \\
\hline $16-S-33$ & 28-Ni-64 & 38-Sr-90 & 47-Ag-107 & 52-Te-129m & 58-Ce-142 & 64-G & 80-Hg-196 & 92-U -239 & 98-Cf-246 \\
\hline $16-S-34$ & 29-Cu-63 & $39-Y-89$ & 47-Ag-109 & 52-Te-130 & 58-Ce-143 & $04-G$ & 80-Hg-198 & $92-U-240$ & 98-Cf-248 \\
\hline $16-S-36$ & $29-\mathrm{Cu}-65$ & 39-Y -90 & 47-Ag-110m & 52-Te-132 & $\begin{array}{l}58-\mathrm{Ce}-143 \\
58-\mathrm{Ce}-144\end{array}$ & 65-1b-159 & 80-Hg-199 & $92-U-241$ & 98-Cf-249 \\
\hline 17-Cl-35 & $30-Z n-64$ & 39-Y -91 & 47-Ag-111 & 53-I -127 & $\begin{array}{l}\text { 50-Ce-144 } \\
59-P r-141\end{array}$ & 65-1D-160 & 80-Hg-200 & 93-Np-234 & 98-Cf-250 \\
\hline 17-Cl-37 & $30-Z n-65$ & $40-Z r-90$ & 48-Cd-106 & 53-I -129 & 59-Pr-142 & $\begin{array}{l}00 \\
66\end{array}$ & 80-Hg-201 & 93-Np-235 & 98-Cf-251 \\
\hline 18-Ar-36 & $30-\mathrm{Zn}-66$ & 40-Zr-91 & 48-Cd-108 & 53-I -130 & 59-Pr-143 & 00 & 80-Hg-202 & 93-Np-236 & 98-Cf-252 \\
\hline 18-Ar-38 & $30-\mathrm{Zn}-67$ & $40-\mathrm{Zr}-92$ & 48-Cd-110 & 53-I -131 & $\begin{array}{l}39-1 \mathrm{r}-143 \\
60-\mathrm{Nd}-142\end{array}$ & $00-1 y-100$ & 80-Hg-204 & 93-Np-237 & 98-Cf-253 \\
\hline 18-Ar-40 & $30-Z n-68$ & $40-Z r-93$ & 48-Cd-111 & 53-I -135 & $\begin{array}{l}60-N d-142 \\
60-N d-143\end{array}$ & $66-10 y-161$ & 81-Tl-203 & 93-Np-238 & 98-Cf-254 \\
\hline $19-K-39$ & $30-Z n-70$ & $40-Z r-94$ & 48-Cd-112 & $54-X e-123$ & $\begin{array}{l}60-N d-143 \\
60-N d-144\end{array}$ & 66-Dy-162 & 81-Tl-205 & 93-Np-239 & 99-Es-251 \\
\hline $19-K-40$ & 31-Ga-69 & $40-Z r-95$ & & 54-Xe-124 & $\begin{array}{l}60-1 \\
60-1\end{array}$ & $66-1$ & 82-Pb-204 & 94-Pu-236 & 99-Es-252 \\
\hline $19-\mathrm{K}-41$ & 31-Ga-71 & & & & & & 82-Pb-206 & 94-Pu-237 & 99-Es-253 \\
\hline $\begin{array}{l}19-\mathrm{h}-41 \\
20-\mathrm{Ca}-40\end{array}$ & $\begin{array}{l}\text { 31-Ga-71 } \\
\text { 32-Ge-70 }\end{array}$ & $\begin{array}{l}40-Z r-96 \\
41-N b-93\end{array}$ & $\begin{array}{l}\text { 48-Cd-114 } \\
\text { 48-Cd-115m }\end{array}$ & $\begin{array}{l}54-X e-126 \\
54-X e-128\end{array}$ & $\begin{array}{l}\text { 60-Nd-146 } \\
60-N d-147\end{array}$ & $\begin{array}{l}6 /-H 0-165 \\
67-H o-166 m\end{array}$ & 82-Pb-207 & 94-Pu-238 & 99-Es-254 \\
\hline $20-\mathrm{Ca}-42$ & 32-Ge-72 & 41-Nb-94 & 48-Cd-116 & $54-X e-129$ & $\begin{array}{l}00-1 \mathrm{Na}-14 / \\
60-\mathrm{Nd}-148\end{array}$ & $\begin{array}{c}\text { 6/-Ho-166m } \\
68-E r-162\end{array}$ & 82-Pb-208 & 94-Pu-239 & 99-Es-254m \\
\hline 20-Ca-43 & 32-Ge-73 & 41-Nb-95 & 49-In-113 & 54-Хe-130 & $60-N d-150$ & 68-Er-164 & 83-Bi-209 & 94-Pu-240 & 99-Es-255 \\
\hline & & & & & & OO-ET-104 & 88-Ra-223 & 94-Pu-241 & 100-Fm-255 \\
\hline
\end{tabular}

\section{PREPRO 2012 Codes}

In addition to the changes in the ENDF/B-VII.1 evaluations, it should be noted that between the last version of this report, where the PREPRO 2010 codes were used, and the current version, where the PREPRO 2012 codes were used, there have been improvements in the ENDF/B Preprocessing codes (PREPRO). The improvements were both in terms of improving the basic methods used by the codes and in terms of incorporating the latest ENDF-6 Formats and Procedures used by the current evaluations. The result is more accurate cross section data throughout the POINT 2012 library.

WARNING - due to recent changes in ENDF-6 Formats and Procedures only the latest version of the ENDF/B Pre-processing codes, namely PREPRO 2012, can be used to accurately process all current ENDF/B-VII evaluations. If you fail to heed this warning and you use any earlier versions of these codes the results will be inaccurate.

The PREPRO 2012 codes run on virtually any computer, and will soon be available FREE on- 
line from the Nuclear Data Section, IAEA, Vienna, Austria, website at,

http://www-nds.iaea.or.at/ndspub/endf/prepro/

\section{Data Processing}

As distributed the original evaluated data includes cross sections represented in the form of a combination of resonance parameters and/or tabulated energy dependent cross sections, nominally at 0 Kelvin temperature. For use in applications, this data has been processed using the 2012 version of the ENDF/B Pre-processing codes (PREPRO 2012) to produce temperature dependent, linearly interpolable in energy, tabulated cross sections, in the ENDF-6 format.

For use in applications this library has been processed into the form of temperature dependent cross sections at eight neutron reactor like temperatures, between 0 and 2100 Kelvin, in steps of 300 Kelvin (the exception being 293.6 Kelvin, for exact room temperature at 20 Celsius). It has also been processed to five astrophysics like temperatures, $1,10,100 \mathrm{eV}, 1$ and $10 \mathrm{keV}$. For reference purposes, 300 Kelvin is approximately $1 / 40 \mathrm{eV}$, so that $1 \mathrm{eV}$ is approximately 12,000 Kelvin. At each temperature the cross sections are tabulated and linearly interpolable in energy.

The steps required and codes used to produce room temperature, linearly interpolable tabulated cross sections, in the ENDF-6 format, are described below; the name of each code in given in parenthesis; for details of each code see reference [R3].

Here are the steps, and PREPRO 2012 codes, used to process the data, in the order in which the codes were used.

1) Linearly interpolable, tabulated cross sections (LINEAR)

2) Including the resonance contribution (RECENT)

3) Doppler broaden all cross sections to temperature (SIGMA1)

4) Check data, define redundant cross sections by summation (FIXUP)

5) Update evaluation dictionary in MF/MT=1/451 (DICTIN)

For the "cold" (0 Kelvin) data steps 1), 2) and 4), 5) were used (no Doppler broadening). For the data at other temperatures, after steps 1) and 2), the data was Doppler broadened to each temperature using step 3), and the results were then made consistent with the ENDF/B formats and conventions using steps 4) and 5), to produce the final distributed data.

The result is linearly interpolable in energy, tabulated, temperature dependent cross sections, in the ENDF-6 format, ready to be used in applications.

Note - this processing only involved the energy dependent neutron cross sections. All other data in the evaluations, e.g., angular and energy distributions, was not affected by this processing, and is identical in all versions of the final results, i.e., it is the same in all of the directories, ORIGINAL, as well as K0 through K2100, and 1ev through 10kev, on the DVDs. 


\section{Accuracy of Results}

Each of the codes described above that was used to process data to obtain tabulated, linearly interpolable in energy cross sections, processed the data to within a user defined accuracy, or allowable uncertainty. The ENDF/B Pre-processing codes (PREPRO 2012) are selfdocumenting, in the sense that the ENDF/B formatted output data that each code produces includes comments at the beginning of each evaluation defining the accuracy to which the cross sections were calculated. The combination of comments added by all of the codes defines the sequence and accuracy used by all of them. The accuracy is the same for all evaluations. Therefore, for exact details of the accuracy of the data, see the comments at the beginning of any evaluation. For use in POINT 2012 all cross sections were reconstructed to within an accuracy of $0.01 \%$ in the thermal range, and $0.1 \%$ at all other energies and temperatures; this is beyond the accuracy to which this data in known, so that I assume that the data processing does not add any significant additional error to the inherent error of the data.

\section{Contents of the Library}

This library contains all of the evaluations in the ENDF/B-VI.1 general purpose library. The below table summarizes the contents of the ENDF/B-VII.1 general purpose library. This library contains evaluations for 423 materials (isotopes or naturally occurring elemental mixtures of isotopes).

This library does not contain data from special purpose ENDF/B-VII libraries, such as fission products, thermal scattering, photon interaction data. To obtain any of these special purpose libraries contact the National Nuclear Data Center, Brookhaven National Laboratory,

\section{ENDF@bnlnd2.dne.bnl.gov}

In the POINT 2012 library each evaluation is stored as a separate file. The following table defines each material and the corresponding filename. The entire library is in the computer independent ENDF-6 character format, which allows the data to be easily transported between computers. The entire library requires approximately 15 gigabyte of storage and is distributed on one DVD compressed; see below for details of the DVD.

This library contains data for some metastable materials, which are indicated by an "M" at the end of their descriptions.

The majority of these evaluations are complete, in the sense that they include all cross sections over the energy range $10^{-5} \mathrm{eV}$ to at least $20 \mathrm{MeV}$. See the appendix for a list of all evaluations, plus a separate list of incomplete evaluations; there are now only a few.

The DVD is compressed; when uncompressed you will find a single directory named POINT2012 containing fifteen (15) sub-directories,

DOCUMENT - A copy of this report in MSWord and PDF formats.

ORIGINAL - The original ENDF/B data before it was processed.

K0 $\quad-0$ Kelvin cross sections 


$\begin{array}{ll}\text { K293.6 } & -293.6 \text { Kelvin cross sections } \\ \text { K600 } & -600 \text { Kelvin cross sections } \\ \text { K900 } & -900 \text { Kelvin cross sections } \\ \text { K1200 } & -1200 \text { Kelvin cross sections } \\ \text { K1500 } & -1500 \text { Kelvin cross sections } \\ \text { K1800 } & -1800 \text { Kelvin cross sections } \\ \text { K2100 } & -2100 \text { Kelvin cross sections } \\ 1 \mathrm{eV} & -1 \mathrm{eV} \text { cross sections } \\ 10 \mathrm{eV} & -10 \mathrm{eV} \text { cross sections } \\ 100 \mathrm{eV} & -100 \mathrm{eV} \text { cross sections } \\ 1 \mathrm{keV} & -1 \mathrm{keV} \text { cross sections } \\ 10 \mathrm{keV} & -10 \mathrm{keV} \text { cross sections }\end{array}$

With the exception of DOCUMENT, each of these directories contains 424 files, one file for each of the 423 evaluation, plus one HTML file to allow interactive data retrieval. Each evaluation is a complete ENDF/B "tape" [R2], including a starting "tape" identification line, and ending with a "tape" end line [R2]. In this form, each file can be used by a wide variety of available computer codes that treat data in the ENDF/B format, e.g., all of the PREPRO codes.

\section{Requesting POINT 2012 Data}

Please do not contact the author of this report to request this data; I do not have the resources necessary to directly respond to requests for this data. This data has been distributed and is Internationally available from nuclear data/code centers throughout the World,

1) Within the United States: contact the National Nuclear Data Center, Brookhaven National Laboratory, Mike Herman at, services@bnlnd2.dne.bnl.gov

2) Within Western Europe: contact the OECD Nuclear Energy Agency/ Data Bank (NEA/DB), Paris, France, programs@nea.fr

3) Otherwise: contact the Nuclear Data Section, International Atomic Energy Agency, Vienna, Austria, Alberto Mengoni at, A.Mengoni@iaea.org 
I recommend that you,

1) Copy the single file from the POINT 2012 DVD to your computer,

2) Uncompress and un-tar the file; then delete the compressed and tar files.

3) You should then have one directory named POINT2012 containing all of the data

4) To random access the data execute (double click) POINT2012.htm.

The main POINT2012 directory will contain the fifteen (15) sub-directories, described above. These POINT 2012 directories include HTML routines to allow interactive retrieval of the data. The result will be a directory of about 15 gigabytes. To put that in perspective, today it costs less than $\$ 0.10$ U.S. to purchase, install, and maintain on-line one gigabyte of disk storage. Therefore the cost of maintaining this 16 gigabyte library on-line is trivial.

\section{Acknowledgments}

I thank Said Mughabghab for his detailed explanation of the use of his newly published resonance parameters [R4] in ENDF/B-VII.0 evaluations. I thank Ramon E. Arcilla, Jr., of the National Nuclear Data Center (NNDC), Brookhaven National Laboratory, for supplying the original ENDF/B-VII.0, used in this project. I thank Liam Costello, of the Nuclear Data Section, International Atomic Energy Agency, for supplying the ENDF/B Pre-processing codes, PREPRO 2012, used in this project. I thank Janice Arwood (RSICC, Oak Rdige) for carefully checking and recommending improvements. I thank Nancy Larsen, Bob MacFarlane, Maurice Greene, and Mike Dunn, for their comparison of their cross section processing codes (SAMMY, NJOY and AMPX) against the PREPRO codes. These comparisons have led to significant improvements in the accuracy and reliability of the results produced by all four codes (SAMMY, NJOY, AMPX, PREPRO). I thank Andre Trkov, Skip Kahler, Robert MacFarlane, Mike Herman and Dave Heinrichs for proofreading the draft of this report and making many helpful corrections and improvements, which I incorporated in the final report. 


\section{References}

[R1a] "POINT 2011: A Temperature Dependent ENDF/B-VII.0 data Cross Section Library", Lawrence Livermore National Laboratory, UCRL-TR-479947, rev. 1, May 2011,

[R1b] "POINT 2009: A Temperature Dependent ENDF/B-VII.0 data Cross Section Library, June 6, 2009.

[R1c] "POINT 2007: A Temperature Dependent ENDF/B-VII.0 data Cross Section Library", Lawrence Livermore National Laboratory, UCRL-TR-228089, February 2007.

[R2] Data Formats and Procedures for the Evaluated Nuclear Data File ENDF-6, BNL-NCS44945, Rev. 11/95, edited by V. McLane, et al. National Nuclear Data Center, Brookhaven National Lab. http://www.nndc.bnl.gov/nndescr/documents/endf/endf102/

[R3] "PREPRO 2010: The 2010 ENDF/B Pre-Processing Codes," by D.E. Cullen, Nuclear Data Section, International Atomic Energy Agency, Vienna, Austria, IAEA-NDS-39, Rev. 14, Oct. 31, 2010; PREPRO 2010 is now publicly available. http://www-nds.iaea.or.at/ndspub/endf/prepro/

PREPRO 2012 will be released for public use early in 2012; until then I recommend you use currently available PREPRO 2010 codes.

[R4] "Atlas of Nuclear Resonances", by S.F. Mughabghab, National Nuclear Data Center, Brookhaven National Laboratory, published by Elsevier, March 2006.

[R5] "Exact Doppler Broadening of Tabulated Cross Sections," by D.E. Cullen and C.R. Weisbin, Nuclear Science and Engineering 60, p. 199 (1975)

[R6] "THERMAL: A Routine Designed to Calculate Neutron Thermal Scattering," by D.E. Cullen, Lawrence Livermore National Laboratory, UCRL-ID-120560-Rev-1, Sept. 1995.

http://home.comcast.net/ redcullen1

[R7] "Verification of High Temperature Free Atom Thermal Scattering in MERCURY Compared to TART", by D.E. Cullen, Scott McKinley and Christian Hagmann, Lawrence Livermore National Laboratory, UCRL-TR-226340, August 1, 2006.

[R8] "TART2005: A Coupled Neutron-Photon 3-D, Time Dependent, Combinatorial Geometry Monte Carlo Transport Code," by D.E. Cullen, Lawrence Livermore National Laboratory, UCRL-SM-218009, Nov. 22, 2005. 
Appendix A: Contents of ENDF/B-VII.1 (32 new + 391 old = 423 total evaluations)

\begin{tabular}{|c|c|c|c|c|c|c|c|c|c|}
\hline $1-H-1$ & 20-Ca-44 & 32-Ge-74 & 42-Мо-92 & 49-In-115 & 54-Хe-131 & 61-Pm-14 & 68-Er-166 & 88-Ra-224 & 94-Pu-242 \\
\hline 1-H -2 & 20-Ca-46 & 32-Ge-76 & 42-M0-94 & Sn-112 & (2) 132 & & 68-Er-167 & 88-Ra-225 & 94-Pu-243 \\
\hline $1-H-3$ & $\begin{array}{l}20-\mathrm{Ca}-40 \\
20-\mathrm{Ca}-48\end{array}$ & $\begin{array}{l}\text { D2-Ge-/0 } \\
33-A s-74\end{array}$ & 42-1010-94 & $\begin{array}{l}50-5 n-112 \\
50-S_{-113}\end{array}$ & 54-Xe-152 & 61-Pm-14 & 68-Er-168 & 88-Ra-226 & 94-Pu-244 \\
\hline $2-\mathrm{He}-3$ & 21-Sc-45 & $\begin{array}{l}\text { 33-As- } 14 \\
\text { 33-As-75 }\end{array}$ & 42-110-93 & $50-5 n-115$ & $54-x e-133$ & 61-Pm-148m & 68-Er-170 & 89-Ac-225 & 94-Pu-246 \\
\hline 2-He-4 & 22-Ti-46 & $\begin{array}{l}\text { 53-As-15 } \\
34-S e-74\end{array}$ & $42-1110-96$ & 50-Sn-114 & $54-X \mathrm{e}-134$ & 61-Pm-149 & 69-Tm-168 & 89-Ac-226 & 95-Am-240 \\
\hline 3-Li-6 & & $34-\mathrm{S}$ & 42-Мо-97 & 50-Sn-115 & 35 & 61-Pm- & 69-Tm-169 & 89-Ac-227 & 95-Am-241 \\
\hline 3-Li-7 & 22-Ti-47 & 34-Se-76 & 42-Мо-98 & 50-Sn-116 & 54-Хe-136 & 62-Sm-144 & 69-Tm-170 & 90-Th-227 & 95-Am-242 \\
\hline 3-Li-7 & 22-Ti-48 & 34-Se-77 & 42-Мo-99 & 50-Sn-117 & 55-Cs-133 & 62-Sm-147 & & h-228 & \\
\hline 4-Be-7 & 22-Ti-49 & 34-Se-78 & 42-Мo-100 & 50-Sn-118 & 55-Cs-134 & 62-Sm-148 & & & \\
\hline 4-Be-9 & 22-Ti-50 & 34-Se-79 & 43-Тc-99 & 50-Sn-119 & 55-Cs-135 & 62-Sm-149 & & 9 & 43 \\
\hline $5-B-10$ & $23-V-50$ & 34-Se-80 & 44-Ru-96 & 50-Sn-120 & 55-Cs-136 & 62-Sm-150 & 4 & 0 & $\mathrm{~m}-244$ \\
\hline $5-B-11$ & $23-V-51$ & 34-Se-82 & 44-Ru-98 & 50-Sn-122 & 55-Cs-137 & 62-Sm-151 & 76 & 90-Th-231 & 95-Am-244m \\
\hline 6-C -Nat & 24-Cr-50 & 35-Br-79 & 44-Ru-99 & $50-S n-123$ & 56-Ba-130 & 62-Sm-152 & 72-Hf-177 & 90-Th-232 & 96-Cm-240 \\
\hline $7-\mathrm{N}-14$ & 24-Cr-52 & $35-\mathrm{Br}-81$ & 44-Ru-100 & $50-S n-124$ & $56-B a-132$ & $62-\mathrm{Sm}_{-153}$ & 72-Hf-178 & 90-Th-233 & 96-Cm-241 \\
\hline 7-N -15 & 24-Cr-53 & $36-\mathrm{Kr}-78$ & $44-R_{1}-101$ & $50-S_{-125}$ & 56-Da-1J2 & $62-3 m-153$ & 72-Hf-179 & 90-Th-234 & 96-Cm-242 \\
\hline 8-0-16 & 24-Cr-54 & $36-\mathrm{Kr}-80$ & 4 & -50 & 50-Da-15J & 62-Sm-154 & 72-Hf-180 & 91-Ра-229 & 96-Cm-243 \\
\hline 8-O - 17 & 25-Mn-55 & $36-\mathrm{Kr}-82$ & $4+-101-102$ & 20 & 56-1а-134 & 63 & 73-Тa-180 & 91-Pa-230 & 96-Cm-244 \\
\hline $9-F-19$ & $26-50-54$ & 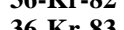 & 44-Ru-103 & 51-Sb-121 & 56-Ba-135 & 63-Eu-152 & 73 & 91-Pa-231 & 96-Cm-245 \\
\hline & & $36-K r-83$ & 44-Ru-104 & 51-Sb-123 & 56-Ba-136 & 63-Eu-153 & & 91-Pa-232 & \\
\hline 11-Na-22 & $26-\mathrm{Fe}-56$ & $36-K r-84$ & 44-Ru-105 & 51-Sb-124 & 56-Ba-137 & 63-Eu-154 & & -Pa-233 & \\
\hline 11-Na-23 & 26-Fe-57 & $36-\mathrm{Kr}-85$ & 44-Ru-106 & 51-Sb-125 & 56-Ba-138 & 63-Eu-155 & & $19-233$ & \\
\hline 12-Mg-24 & 26-Fe-58 & $36-\mathrm{Kr}-86$ & 45-Rh-103 & 51-Sb-126 & 56-Ba-140 & 63-Eu-156 & 182 & 30 & 48 \\
\hline 12-Mg-25 & 27-Co-58 & 37-Rb-85 & 45-Rh-105 & 52-Te-120 & 57-La-138 & 63-Eu-157 & 183 & 92-U -231 & 96-Cm-249 \\
\hline 12-Mg-26 & 27-Co-58m & 37-Rb-86 & 46-Pd-102 & 52-Te-122 & 57-La-139 & 64-Gd-152 & -184 & 92-U -232 & 96-Cm-250 \\
\hline 13-Al-27 & 27-Co-59 & 37-Rb-87 & 46-Pd-104 & 52-Te-123 & 57-La-140 & 64-Gd-153 & 74-W -186 & 92-U -233 & 97-Bk-245 \\
\hline 14-Si-28 & 28-Ni-58 & 38-Sr-84 & 46-Pd-105 & 52-Te-124 & 58-Ce-136 & 64-Gd-154 & 75-Re-185 & 92-U -234 & 97-Bk-246 \\
\hline 14-Si-29 & 28-Ni-59 & 38-Sr-86 & 46-Pd-106 & 52-Te-125 & 58-Ce-138 & & 75-Re-187 & $92-U-235$ & 97-Bk-247 \\
\hline 14-Si-30 & $28-\mathrm{Ni}-60$ & 38-Sr-87 & 46-Pd-107 & 52-Te-126 & 58-Ce-139 & & 77-Ir-191 & $92-U-236$ & 97-Bk-248 \\
\hline 15-P-31 & 28-Ni-61 & 38-Sr-88 & 46-Pd-108 & 52-Te-127m & $\begin{array}{l}\text { 5o-ce-159 } \\
58-C e-140\end{array}$ & 64 & 77-Ir-193 & 92-U -237 & 97-Bk-249 \\
\hline $16-S-32$ & 28-Ni-62 & $38-S r-89$ & 46-Pd-110 & 52-Te-128 & 58-Ce-140 & 64-Gd-157 & 79-Au-197 & 92-U -238 & 97-Bk-250 \\
\hline $16-5-33$ & $28-\mathrm{Ni}-64$ & $38-S r=90$ & (7) 107 & 52 T120 & 58-ce-141 & 64-Gd-158 & 80-Hg-196 & 92-U -239 & 98-Cf-246 \\
\hline $16-34$ & $50-1$ & 50 - & 4/-Ag-10/ & -12911 & $58-\mathrm{Ce}-142$ & 64-Gd-160 & 80-Hg-198 & $92-U-240$ & 98-Cf-248 \\
\hline $10-5-34$ & $29-C$ & $39-1$ & 47-Ag-109 & 52-T & $58-C$ & 65-Tl & 80-Hg-199 & $92-U-241$ & 98-Cf-249 \\
\hline $16-S-36$ & 29-Cu-65 & 39-Y -90 & 47-Ag-110m & 52-Te-132 & 58-Сe-144 & 65-Tb-160 & $80-\mathrm{Co}_{-} 200$ & 93-Nn-234 & 98-Cf-250 \\
\hline 17-Cl-35 & $30-Z n-64$ & 39-Y -91 & 47-Ag-111 & 53-I -127 & 59-Pr-141 & 66-Dy-156 & & & \\
\hline 17-Cl-37 & $30-\mathrm{Zn}-65$ & 40-Zr-90 & 48-Cd-106 & 53-I -129 & 59-Pr-142 & 66-Dy-158 & & & \\
\hline 18-Ar-36 & $30-\mathrm{Zn}-66$ & 40-Zr-91 & 48-Cd-108 & 53-I -130 & 59-Pr-143 & 66-Dy-160 & & & \\
\hline 18-Ar-38 & $30-\mathrm{Zn}-67$ & 40-Zr-92 & 48-Cd-110 & 53-I -131 & $60-\mathrm{Nd}-142$ & 66-Dy-161 & 04 & 93-Np-237 & 98-Cf- \\
\hline 18-Ar-40 & $30-\mathrm{Zn}-68$ & $40-Z r-93$ & 48-Cd-111 & 53-I -135 & $60-N d-143$ & 66-Dy-162 & 81 & 93-Np-238 & 98-Cf-254 \\
\hline $19-K-39$ & $30-\mathrm{Zn}-70$ & $40-Z r-94$ & 48-Cd-112 & $54-X e-123$ & $60-N d-144$ & 66-Dy-163 & 81-Tl-205 & 93-Np-239 & 99-Es-251 \\
\hline $19-\mathrm{K}-40$ & 31-Ga-69 & 40-Zr-95 & 48-Cd-113 & $54-X e-124$ & $60-N d-145$ & 66-Dy-164 & 82-Pb-204 & 94-Pu-236 & 99-Es-252 \\
\hline $19-K-41$ & 31-Ga-71 & 40-Zr-96 & 48-Cd-114 & 54-Хe-126 & $60-N d-146$ & $67-H 0-165$ & 82-Pb-206 & 94-Pu-237 & 99-Es-253 \\
\hline 20-Ca-40 & 32-Ge-70 & 41-Nb-93 & 48-Cd-115m & 54-Хe-128 & $60-\mathrm{n}$ & $67-\mathrm{H}$ & 82-Pb-207 & 94-Pu-238 & 99-Es-254 \\
\hline 20-Ca-42 & 32-Ge-72 & 41-Nb-94 & 48-Cd-116 & 54-Хе-129 & $60-\mathrm{Nd}-148$ & 68-Er-162 & 82-Pb-208 & 94-Pu-239 & 99-Es-254m \\
\hline 20-Ca-43 & 32-Ge-73 & 41-Nb-95 & 49-In-113 & $54-X e-130$ & $60-N d-150$ & 68-Er-164 & 83-Bi-209 & 94-Pu-240 & 99-Es-255 \\
\hline & & & & $34-\lambda \mathrm{e}-100$ & $00-1 \times 0-150$ & 08-Er-104 & 88-Ra-223 & 94-Pu-241 & 100-Fm-255 \\
\hline
\end{tabular}




\section{Appendix B: Elemental vs. Isotopic Evaluations}

Successive versions of ENDF/B have replaced elemental evaluations by isotopic evaluations. Between ENDF/B-VI and VII 13 elemental evaluations were deleted, i.e., included in ENDF/BVI, but not included in ENDF/B-VII, with VII.0 only including elemental evaluations for three elements: 6-C, 23-V, and 30-Zn. Between VII.0 and VII.1 2 elemental evaluations were deleted (23-V, and 30-Zn), and replaced by isotopic evaluations, leaving only 6-C: 6-C-12 98.93\%/ 6-C$131.07 \%$ missing - so that $6 \mathrm{C}-\mathrm{Nat}$ is almost entirely the single isotope 6-C-12.

In addition evaluations for the isotopes of $68-\mathrm{Tm}$ and $81-\mathrm{Tl}$ have been added.

All of these isotopes in VII.1 are complete, in the sense that they include major cross sections (elastic, capture, inelastic) over the energy range $10^{-5} \mathrm{eV}$ up to at least $20 \mathrm{MeV}$.

WARNING: Be aware that evaluating isotopes is difficult and the quality of minor isotopes may be poor. To my knowledge as yet the summing these isotopes to define equivalent elemental evaluations has not been verified against experimental measurements.

New Isotopic evaluations in VII.1 (28 new, 19 old)

\begin{tabular}{|c|c|c|c|c|c|}
\hline Element & Isotope & Element & Isotope & Element & Isotope \\
\hline \multirow[t]{3}{*}{ 12-Mg-Nat } & $12-\mathrm{Mg}-24$ & \multirow[t]{5}{*}{ 22-Ti-Nat } & $22-\mathrm{Ti}-46$ & \multirow[t]{8}{*}{ 42-Mo-Nat } & $42-\mathrm{Mo}-92$ \\
\hline & $12-\mathrm{Mg}-25$ & & $22-\mathrm{Ti}-47$ & & $42-\mathrm{Mo}-94$ \\
\hline & $12-\mathrm{Mg}-26$ & & $22-\mathrm{Ti}-48$ & & 42-Mo- 95 \\
\hline \multirow[t]{3}{*}{ 14-Si-Nat } & $14-S i-28$ & & 22-Ti- 49 & & $42-\mathrm{Mo}-96$ \\
\hline & $14-\mathrm{Si}-29$ & & $22-\mathrm{Ti}-50$ & & 42-Mo- 97 \\
\hline & $14-\mathrm{Si}-30$ & \multirow[t]{2}{*}{$23-\mathrm{V}-\mathrm{Nat}$} & $23-v-50$ & & $42-\mathrm{Mo}-98$ \\
\hline \multirow[t]{4}{*}{$16-\mathrm{S}-\mathrm{Nat}$} & $16-S-32$ & & $23-v-51$ & & 42-Mo- 99 \\
\hline & $16-s-33$ & \multirow[t]{6}{*}{$30-\mathrm{Zn}-\mathrm{Nat}$} & $30-\mathrm{Zn}-64$ & & $42-\mathrm{Mo}-100$ \\
\hline & $16-s-34$ & & $30-\mathrm{Zn}-65$ & \multirow[t]{5}{*}{ 49-In-Nat } & 49-In-113 \\
\hline & $16-s-36$ & & $30-\mathrm{Zn}-66$ & & $49-I n-115$ \\
\hline \multirow{2}{*}{ 17-Cl-Nat } & $17-\mathrm{Cl}-35$ & & $30-\mathrm{Zn}-67$ & & $69-T m-168$ \\
\hline & $17-\mathrm{Cl}-37$ & & $30-\mathrm{Zn}-68$ & & 69-Tm-169 \\
\hline \multirow{3}{*}{ 19-K -Nat } & $19-K-39$ & & $30-\mathrm{Zn}-70$ & & 69-Tm-170 \\
\hline & $19-\mathrm{K}-40$ & \multirow{2}{*}{$31-\mathrm{Ga}-\mathrm{Nat}$} & $31-\mathrm{Ga}-69$ & \multirow{5}{*}{ 72-Hf-Nat } & $72-\mathrm{Hf}-174$ \\
\hline & $19-K-41$ & & $31-\mathrm{Ga}-71$ & & $72-\mathrm{Hf}-176$ \\
\hline \multirow[t]{9}{*}{ 20-Ca-Nat } & $20-\mathrm{Ca}-40$ & \multirow{9}{*}{$40-\mathrm{Zr}-\mathrm{Nat}$} & $40-Z r-90$ & & $72-\mathrm{Hf}-177$ \\
\hline & $20-\mathrm{Ca}-42$ & & $40-Z r-91$ & & $72-\mathrm{Hf}-178$ \\
\hline & $20-\mathrm{Ca}-43$ & & $40-Z r-92$ & & 72-Hf-179 \\
\hline & $20-\mathrm{Ca}-44$ & & $40-Z r-93$ & \multirow{6}{*}{ 74-W -Nat } & $\begin{array}{l}72-\mathrm{H} \pm-180 \\
74-W-182\end{array}$ \\
\hline & $20-\mathrm{Ca}-46$ & & $40-Z r-94$ & & $\begin{array}{l}14-W-182 \\
74-W-183\end{array}$ \\
\hline & $20-\mathrm{Ca}-48$ & & $40-Z r-95$ & & $74-W-184$ \\
\hline & & & $40-Z r-96$ & & $74-W-186$ \\
\hline & & & & & 81-T1-203 \\
\hline & & & & & 81-T1-205 \\
\hline
\end{tabular}




\section{Appendix C: Completeness of VII.1 and VII.0}

Here I present the results of simple tests to check the completeness of VV,1 and VII.0. Compared to the results for VII.0, the VII.1 show great improvement.

The results presented here should not be interpreted as indicating ERRORS, but rather as WARNINGS, that we should check the indicated data; in many cases I judge the data to be o.k. 4-Be-7 is a partial evaluations that failed almost all tests, and should never have been included in a ENDF/B general purpose library. Here I checked,

1) $\mathrm{MT}=2,102,18,4,16$ (elastic, capture, fission, inelastic, $n, 2 \mathrm{n}$ ).

2) $\mathrm{MF}=3,4,5,6$ (cross sections, angular, energy, double differential)

For these test I assume,

1) Every evaluation includes MT $=2,102,4$ and 16; this is obviously not true for some light isotopes, but for completeness they are included here, i.e., this is merely to inform users. Of the heavier isotopes only 28 -Ni-59 appears to be a problem with no inelastic data.

2) All cross sections are positive (>0) above their threshold up to at least $20 \mathrm{MeV}$. This is not true for some reactions which do not extend up to at least $20 \mathrm{MeV}$, particularly high energy capture, which is o.k. There are also a number of cases where the elastic is negative due to the resonance contribution.

3) No isotopes with $\mathrm{Z}<90$ include MT=18 (fission). For VII.0 this test found the obvious ERROR in 43-Tc-99, which has positive fission widths = NONSENSE!!! This was corrected for VII.1. It also flagged isotopes of 88Ra and 89-Ac, which are questionable.

4) I included many more completeness tests, but these were the only ones that failed.

\section{VII.1 Completeness Results}

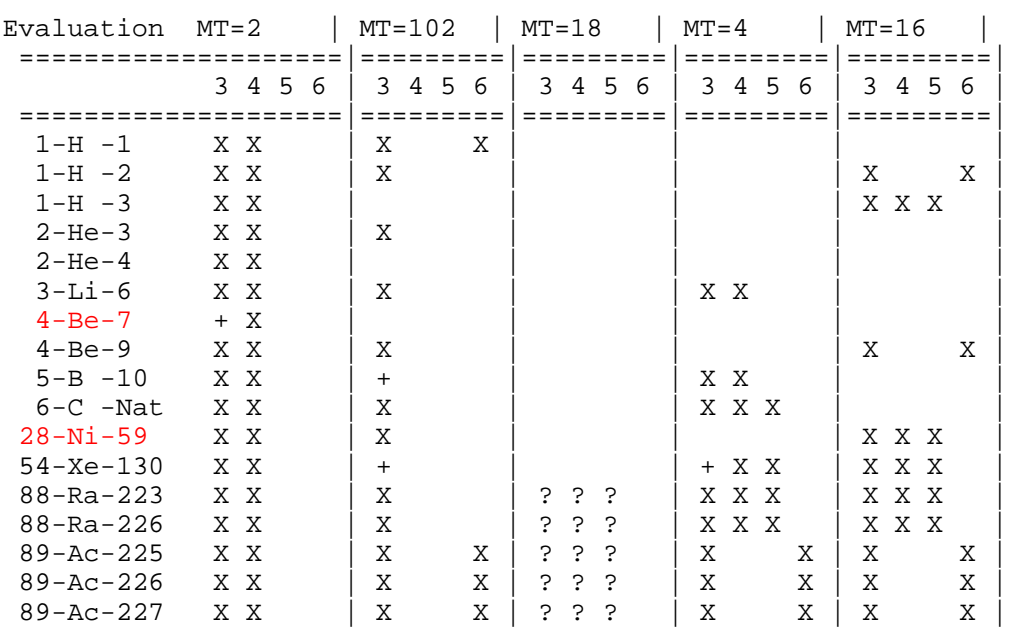

The above rather short list for VII.1 can be compared to the below much longer list for VII.0 to see the improvements in completeness. 
VII.0 Completeness Results

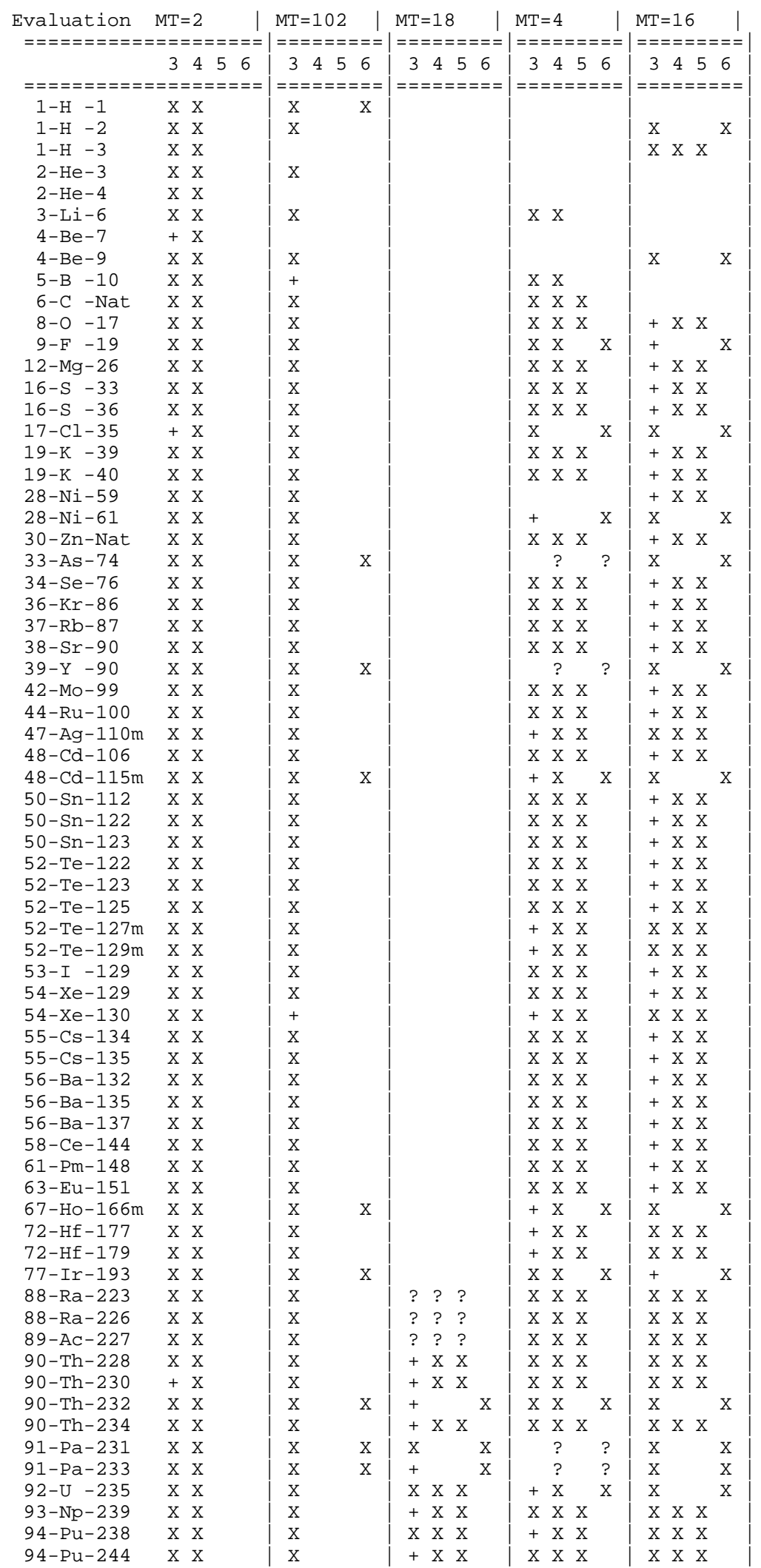




\begin{tabular}{|c|c|c|c|c|c|c|c|}
\hline $94-\mathrm{Pu}-246$ & $\mathrm{X} x$ & $\mathrm{X}$ & $+x x$ & $\mathrm{X} X \mathrm{X}$ & & $\mathrm{X} X$ & \\
\hline $95-A m-242 m$ & $\mathrm{X} X$ & $\mathrm{x}$ & $\mathrm{X} X \mathrm{X}$ & $+x$ & $\mathrm{X}$ & $\mathrm{X}$ & $\mathrm{X}$ \\
\hline $95-A m-244 m$ & $\mathrm{XX}$ & $\mathrm{X}$ & $\mathrm{X} X \mathrm{X}$ & $+\mathrm{XX}$ & & $\mathrm{X} X$ & \\
\hline $96-\mathrm{Cm}-241$ & $\mathrm{XX}$ & + & $\mathrm{X} X \mathrm{X}$ & $+\mathrm{XX}$ & & $\mathrm{X} X$ & \\
\hline $96-\mathrm{Cm}-242$ & $\mathrm{x} x$ & $\mathrm{x}$ & $+\mathrm{XX}$ & $+\mathrm{XX}$ & & $\mathrm{X} X$ & \\
\hline $96-\mathrm{Cm}-248$ & $+x$ & $\mathrm{X}$ & $\mathrm{X} X \mathrm{X}$ & $+\mathrm{XX}$ & & $\mathrm{X} X$ & \\
\hline $98-C f-250$ & $\mathrm{XX}$ & $\mathrm{X}$ & $+\mathrm{XX}$ & $\mathrm{X} X \mathrm{X}$ & & $+x$ & \\
\hline $98-C f-251$ & $\mathrm{Xx}$ & $\mathrm{X}$ & $\mathrm{X} X \mathrm{X}$ & $\mathrm{X} X \mathrm{X}$ & & $+x$ & \\
\hline $98-C f-252$ & $\mathrm{XX}$ & $\mathrm{X}$ & $\mathrm{X} X \mathrm{X}$ & $\mathrm{X} X \mathrm{X}$ & & $+x$ & \\
\hline $98-C f-253$ & $\mathrm{XX}$ & + & $+x x$ & & & & \\
\hline $99-E s-253$ & $\mathrm{X} X$ & + & & & & & \\
\hline
\end{tabular}




\section{Appendix D: Summary of $<v(E)>$ for all isotopes in ENDF/B-VII.1 and VII.0}

For applications involving fission (F) I require both prompt and delayed neutrons per fission. In the ENDF/B format the evaluator can optionally include: Total (T), Delayed (D) and/or Prompt $(\mathrm{P})$; given any two of these three we can uniquely define the third. Below is a summary of all fissile/fertile materials in ENDF/B-VII.1, indicating the neutrons per fission data included for each isotope. This table indicates each evaluation that includes,

$\mathrm{F}=$ Fission cross section $(\mathrm{MF} / \mathrm{MT}=3 / 18)$

$\mathrm{T}=$ Total $\langle v(E)\rangle \quad(\mathrm{MF} / \mathrm{MT}=1 / 452)$

$\mathrm{D}=$ Delayed $\langle v(E)\rangle \quad(\mathrm{MF} / \mathrm{MT}=1 / 455)$

$\mathrm{P}=$ Prompt $\langle v(E)\rangle \quad(\mathrm{MF} / \mathrm{MT}=1 / 456)$

This table indicates VII.1 is in much better shape than VII.0, where more evaluations only included total $(\mathrm{T})<v(E)>$. In addition there are three evaluations that only include total (T) $<v(E)>$ : 88-Ra-233, 88-Ra-226, and 94-Pu-243. It is also questionable whether or not 88-Ra and $89-A c$ should be identified as fissile/fertile.

Summary of all fissile/fertile isotopes in ENDF/B-VII.1 $<v(E)>(85$ evaluations $)$

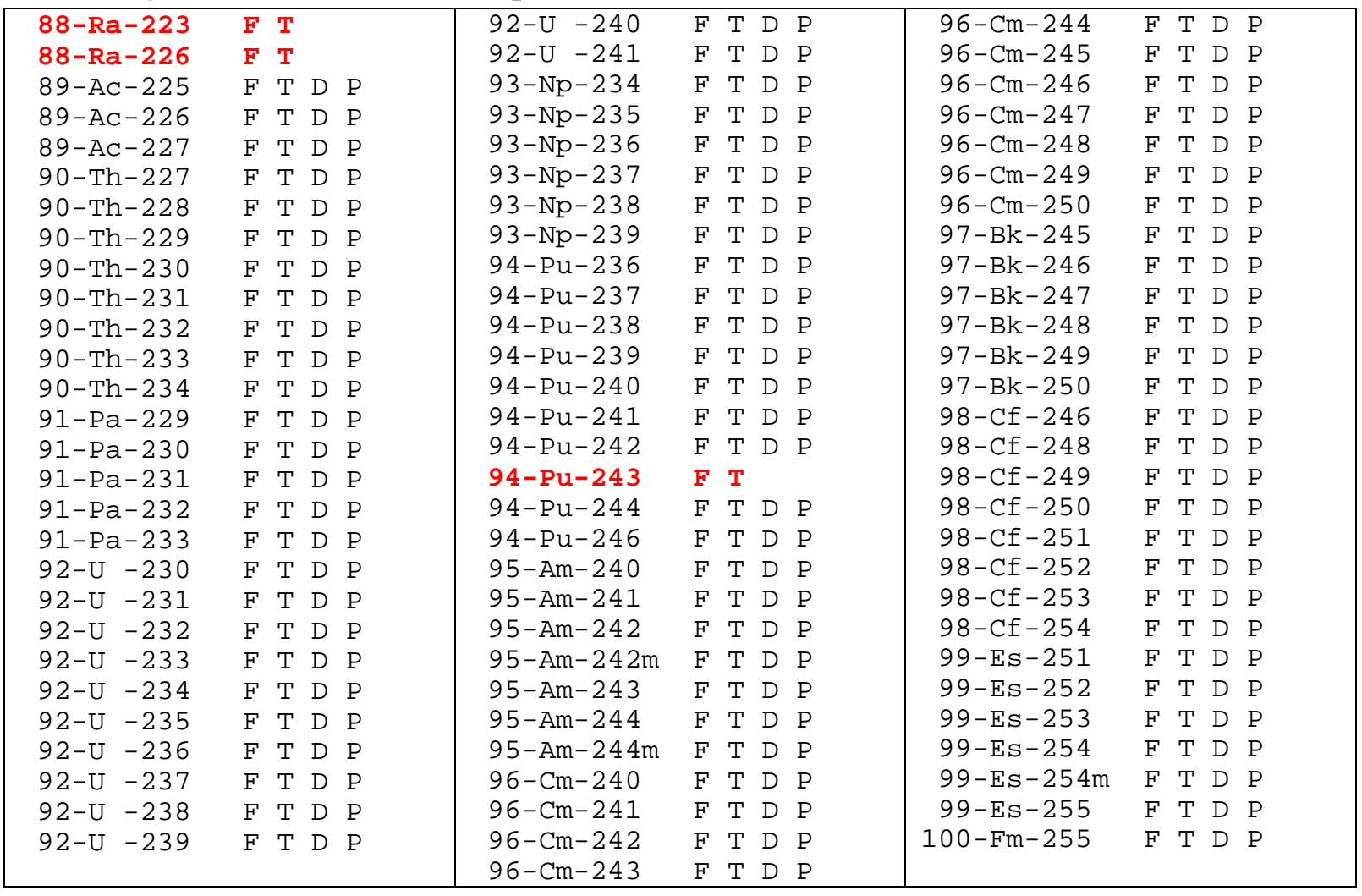




\section{Summary of all fissile/fertile isotopes in ENDF/B-VII.0 $<v(E)>(65$ evaluations $)$}

Comparing the above table for VII.1 and the below table for VII.0 we can see that the $\langle v(E)\rangle$ data is much improved, but VII.1 still has the same three deficiencies that we found in VII.0, namely evaluations that only include total $(\mathrm{T})<v(E)>$ : 88-Ra-233, 88-Ra-226, and 94-Pu-243.

\begin{tabular}{|c|c|c|c|c|c|c|c|c|c|c|c|c|c|}
\hline $88-R a-223$ & $F$ & $\mathrm{~T}$ & & $92-U-241$ & $F$ & $\bar{T}$ & $\overline{D F}$ & & $96-\mathrm{Cm}-241$ & $F$ & & & \\
\hline $88-R a-226$ & $\mathbf{F}$ & $\mathbf{T}$ & & $93-N p-235$ & $\mathrm{~F}$ & $\mathrm{~T}$ & D & $\mathrm{P}$ & $96-\mathrm{Cm}-242$ & $\mathrm{~F}$ & $\mathrm{~T}$ & D & $\mathrm{P}$ \\
\hline $89-A c-227$ & $\mathbf{F}$ & $\mathbf{T}$ & & $93-N p-236$ & $\mathrm{~F}$ & $\mathrm{~T}$ & D $\mathrm{F}$ & $\mathrm{P}$ & $96-\mathrm{Cm}-243$ & $\mathrm{~F}$ & $\mathrm{~T}$ & D & $\mathrm{P}$ \\
\hline $90-T h-227$ & $\mathrm{~F}$ & $\mathrm{~T} D$ & $\mathrm{P}$ & $93-N p-237$ & $\mathrm{~F}$ & $\mathrm{~T}$ & D $\mathrm{F}$ & $\mathrm{P}$ & $96-\mathrm{Cm}-244$ & $\mathrm{~F}$ & $\mathrm{~T}$ & D & $\mathrm{P}$ \\
\hline $90-T h-228$ & $\mathrm{~F}$ & $\mathrm{~T} D$ & $\mathrm{P}$ & $93-N p-238$ & $\mathrm{~F}$ & $\mathrm{~T}$ & D & $\mathrm{P}$ & $96-\mathrm{Cm}-245$ & $\mathrm{~F}$ & $\mathrm{~T}$ & D & $\mathrm{P}$ \\
\hline $90-T h-229$ & $\mathrm{~F}$ & $\mathrm{~T} D$ & $\mathrm{P}$ & $93-N p-239$ & $\mathbf{F}$ & $\mathbf{T}$ & & & $96-C m-246$ & $\mathrm{~F}$ & $\mathrm{~T}$ & D & $\mathrm{P}$ \\
\hline $90-T h-230$ & $\mathbf{F}$ & $\mathbf{T}$ & & $94-P u-236$ & $\mathrm{~F}$ & $\mathrm{~T}$ & D & $\mathrm{P}$ & $96-\mathrm{Cm}-247$ & $\mathrm{~F}$ & $\mathrm{~T}$ & $\mathrm{D}$ & $\mathrm{P}$ \\
\hline $90-T h-232$ & $\mathrm{~F}$ & $\mathrm{~T} \mathrm{D}$ & $\mathrm{P}$ & $94-P u-237$ & $\mathbf{F}$ & $\mathbf{T}$ & & & $96-\mathrm{Cm}-248$ & $\mathbf{F}$ & $\mathbf{T}$ & & \\
\hline $90-T h-233$ & $\mathrm{~F}$ & $\mathrm{~T} \mathrm{D}$ & P & $94-P u-238$ & $\mathrm{~F}$ & $\mathrm{~T}$ & D & $\mathrm{P}$ & $96-\mathrm{Cm}-249$ & $\mathrm{~F}$ & $\mathrm{~T}$ & D & $\mathrm{P}$ \\
\hline $90-T h-234$ & $\mathrm{~F}$ & $\mathrm{~T} \mathrm{D}$ & $\mathrm{P}$ & $94-P u-239$ & $\mathrm{~F}$ & $\mathrm{~T}$ & D & $\mathrm{P}$ & $96-\mathrm{Cm}-250$ & $\mathrm{~F}$ & $\mathrm{~T}$ & D & $\mathrm{P}$ \\
\hline $91-\mathrm{Pa}-231$ & F & $\mathrm{T} \mathrm{D}$ & $\mathrm{P}$ & $94-P u-240$ & $\mathrm{~F}$ & $\mathrm{~T}$ & D & $\mathrm{P}$ & $97-B k-249$ & $\mathrm{~F}$ & $\mathrm{~T}$ & D & $\mathrm{P}$ \\
\hline $91-\mathrm{Pa}-232$ & $\mathrm{~F}$ & $\mathrm{~T} D$ & $\mathrm{P}$ & $94-\mathrm{Pu}-241$ & F & $\mathrm{T}$ & D & $\mathrm{P}$ & $97-B k-250$ & $\mathrm{~F}$ & $\mathrm{~T}$ & D & $\mathrm{P}$ \\
\hline $91-\mathrm{Pa}-233$ & $\mathrm{~F}$ & $\mathrm{~T} D$ & $\mathrm{P}$ & $94-\mathrm{Pu}-242$ & $\mathrm{~F}$ & $\mathrm{~T}$ & D & $\mathrm{P}$ & $98-C f-249$ & $\mathrm{~F}$ & $\mathrm{~T}$ & D & $\mathrm{P}$ \\
\hline $92-U-232$ & $\mathrm{~F}$ & $\mathrm{~T} D$ & $\mathrm{P}$ & $94-P u-243$ & $\mathbf{F}$ & $\mathbf{T}$ & & & $98-C f-250$ & $\mathbf{F}$ & $\mathbf{T}$ & & \\
\hline $92-U-233$ & $\mathrm{~F}$ & $\mathrm{~T} D$ & $\mathrm{P}$ & $94-P u-244$ & $\mathbf{F}$ & $\mathbf{T}$ & & & $98-C f-251$ & $\mathrm{~F}$ & $\mathrm{~T}$ & D & $\mathrm{P}$ \\
\hline $92-U-234$ & F & $\mathrm{T} D$ & $\mathrm{P}$ & $94-\mathrm{Pu}-246$ & $\mathrm{~F}$ & $\mathrm{~T}$ & D & $\mathrm{P}$ & $98-C f-252$ & $\mathrm{~F}$ & $\mathrm{~T}$ & & \\
\hline $92-U-235$ & F & $\mathrm{T} \mathrm{D}$ & $\mathrm{P}$ & $95-A m-241$ & $\mathrm{~F}$ & $\mathrm{~T}$ & D & $\mathrm{P}$ & $98-C f-253$ & $\mathbf{F}$ & $\mathbf{T}$ & & \\
\hline $92-U-236$ & $\mathrm{~F}$ & $\mathrm{~T} D$ & $\mathrm{P}$ & $95-A m-242$ & F & $\mathrm{T}$ & D & $\mathrm{P}$ & $98-C f-254$ & $\mathrm{~F}$ & $\mathrm{~T}$ & $\mathrm{D}$ & $\mathrm{P}$ \\
\hline $92-U-237$ & $\mathrm{~F}$ & $\mathrm{~T} D$ & $\mathrm{P}$ & $95-\mathrm{Am}-242 \mathrm{~m}$ & $\mathrm{~F}$ & $\mathrm{~T}$ & D 1 & $\mathrm{P}$ & $99-E s-254$ & $\mathrm{~F}$ & $\mathrm{~T}$ & D & $\mathrm{P}$ \\
\hline $92-U-238$ & $\mathrm{~F}$ & $\mathrm{~T} \quad \mathrm{D}$ & $\mathrm{P}$ & $95-A m-243$ & $\mathrm{~F}$ & $\mathrm{~T}$ & D 1 & $\mathrm{P}$ & $99-E s-255$ & $\mathrm{~F}$ & $\mathrm{~T}$ & D & P \\
\hline $92-U-239$ & $\mathrm{~F}$ & $\mathrm{~T} D$ & $\mathrm{P}$ & $95-A m-244$ & $\mathrm{~F}$ & $\mathrm{~T}$ & D 1 & $\mathrm{P}$ & $100-\mathrm{Fm}-255$ & $\mathrm{~F}$ & $\mathrm{~T}$ & D & $\mathrm{P}$ \\
\hline $92-U-240$ & $\mathrm{~F}$ & $\mathrm{~T} D$ & $\mathrm{P}$ & $95-A m-244 m$ & $\mathrm{~F}$ & $\mathrm{~T}$ & D 1 & $\mathrm{P}$ & & & & & \\
\hline
\end{tabular}




\section{Appendix E: The Effects of Temperature and Doppler Broadening}

For those readers who are not familiar with the effects of temperature and Doppler broadening on neutron cross sections and transport, for details I suggest that you read references [R5] and [R6], listed below. Here I will give a brief description of these effects. Users of neutron cross sections should be aware that there are several important effects of temperature and Doppler broadening,

1) There is the well known effect in the neutron resonance region, where as the temperature increases resonances become broader, hence the name Doppler broadening. Figure 1 below illustrates the effect of temperature on the $U^{238}$ capture cross section for neutron reactor like temperatures, and figure 2 illustrates this effect for astrophysical like temperatures. These figures each contain four sub-figures, with each sub-figure comparing cross sections at two progressively higher temperatures. In both figure 1 and 2 each sub-figure shows exactly the same energy and cross section range. From these figures we can see that as temperature increases the peaks of the resonances become lower, and the minima between resonances become higher. At extremely high temperature the entire resonance structure disappears and the cross section approaches a simple $1 / \mathrm{v}$ shape (where $\mathrm{v}$ is the neutron speed). This temperature effect will have a very important effect on resonance self-shielding in any neutron transport calculation. You should note from these figures that due to the large resonance spacing in $\mathrm{U}^{\mathbf{2 3 8}}$ the resonance structure can still be seen up to very high temperatures.

To understand the importance of considering temperature we should consider reaction rates, such as captures/second, in various systems. In optically thin systems (few mean free paths dimensions) the flux will be unshielded, and our reaction rates will be defined by a simple cross section average,

Unshielded Capture $=\int_{E 1}^{E 2}[\Sigma c(E) \phi(E)] d E=$ capture cross section times neutron flux

In optically thick systems (many mean free paths dimensions) the flux will be shielded (the flux is suppressed by the total cross section) and our reaction rates must include the effect of selfshielding on the cross section average,

Shielded Capture $=\int_{E 1}^{E 2}[\Sigma c(E) \phi(E) / \Sigma t(E)] d E=$ including one over total cross section

Consider for example the U238 capture cross section between 1 and $10 \mathrm{keV}$ as shown in fig. 1 and 2. If we calculate the unshielded and shielded average capture cross section for the energy interval over the range of temperatures shown in figs. 1 and 2, we obtain the results shown below in table 1.

What we see from these results is that the unshielded average capture cross section is virtually independent of temperature, being about 1 barn over the entire temperature range. In contrast the shielded average cross section varying by over a factor of three between the $0 \mathrm{~K}$ average $(0.293$ 
barns) and the $10 \mathrm{keV}$ average ( 0.939 barns). The point to learn from this is that without including the effect of self-shielding in multi-group calculations, temperature has very little effect on the average cross sections, which is quite simply wrong for optically thick systems.

\begin{tabular}{|c|c|c|}
\hline Temp. & $\begin{array}{l}\text { Unshielded } \\
\text { (barns) }\end{array}$ & $\begin{array}{l}\text { Shielded } \\
\text { (barns) }\end{array}$ \\
\hline $0 \mathrm{~K}$ & 0.996 & 0.293 \\
\hline $293.6 \mathrm{~K}$ & 0.966 & 0.526 \\
\hline $600 \mathrm{~K}$ & 0.996 & 0.576 \\
\hline $1,200 \mathrm{~K}$ & 0.996 & 0.630 \\
\hline $12,000 \mathrm{~K}(1 \mathrm{eV})$ & 0.996 & 0.799 \\
\hline $10 \mathrm{eV}$ & 0.998 & 0.905 \\
\hline $100 \mathrm{eV}$ & 1.000 & 0.933 \\
\hline $1 \mathrm{keV}$ & 1.004 & 0.935 \\
\hline $10 \mathrm{keV}$ & 1.007 & 0.939 \\
\hline
\end{tabular}

2) Another, less well known, effect of Doppler broadening is at lower energies where as temperature increases the low energy constant scattering cross section increases and at very low energies approaches a simple $1 / \mathrm{v}$ shape (where $\mathrm{v}$ is the neutron speed); this effect is explained in detail in ref [R5]. Figure 3 illustrates the effect of temperature on the hydrogen total cross section. From this figure we can see that starting from a "cold" ( 0 Kelvin $)$ cross section that is constant at about 20 barns, as temperature increases the cross section increases. Compared to the "cold" 20 barn cross section, at thermal energy the Doppler broadened cross section is about 30 barns, i.e., $50 \%$ higher. Note also from this figure that this effect extends well above thermal energy. For example, at 293.6 Kelvin the thermal energy is $0.0253 \mathrm{eV}$, but we can see this effect up to about $1 \mathrm{eV}$; a factor of 400 higher in energy. From the lower half of figure 2 we can see that at very low energy the cross section approaches a simple 1/v shape (where $v$ is the neutron speed) and the cross sections at various temperatures become proportional to one another. This effect on the cross sections at low energy is very important for thermal and low energy neutron systems.

3) Yet another important effect of temperature is that at lower energies neutrons do not slow down in energy as quickly and neutron scatter can even result in the upscatter of neutrons, i.e., when neutrons scatter they can gain, rather than lose, energy. This is a well known effect at low energies, where thermal scattering law data or a free gas model is used to model the interaction of neutrons with target atoms that are moving about with thermal motion. Figure 4 illustrates the effect of temperature on the neutron spectrum over a wide range of temperatures [R7]. This effect can also be important at higher energies, particularly near narrow resonances, where thermal motion of the target atoms can cause neutrons to slightly upscatter, but even slight upscatter can cause a neutron to scatter from below to above the energy of a very narrow resonance. See reference [R6], below for a routine designed to be used in conjunction with the SIGMA1 method of Doppler broadening [R5], to handle neutron thermal scattering. This routine [R6] is completely compatible for use with the cross sections included here, since these cross sections were Doppler broadened using the SIGMA1 method [R5]. The combination of SIGMA1 [R5] Doppler broadened cross sections and THERMAL [R6] to handle thermal scattering, is currently used in the TART Monte Carlo transport code [R8]. 
Fig.1: Effect of Doppler Broadening on Resonance Cross Sections
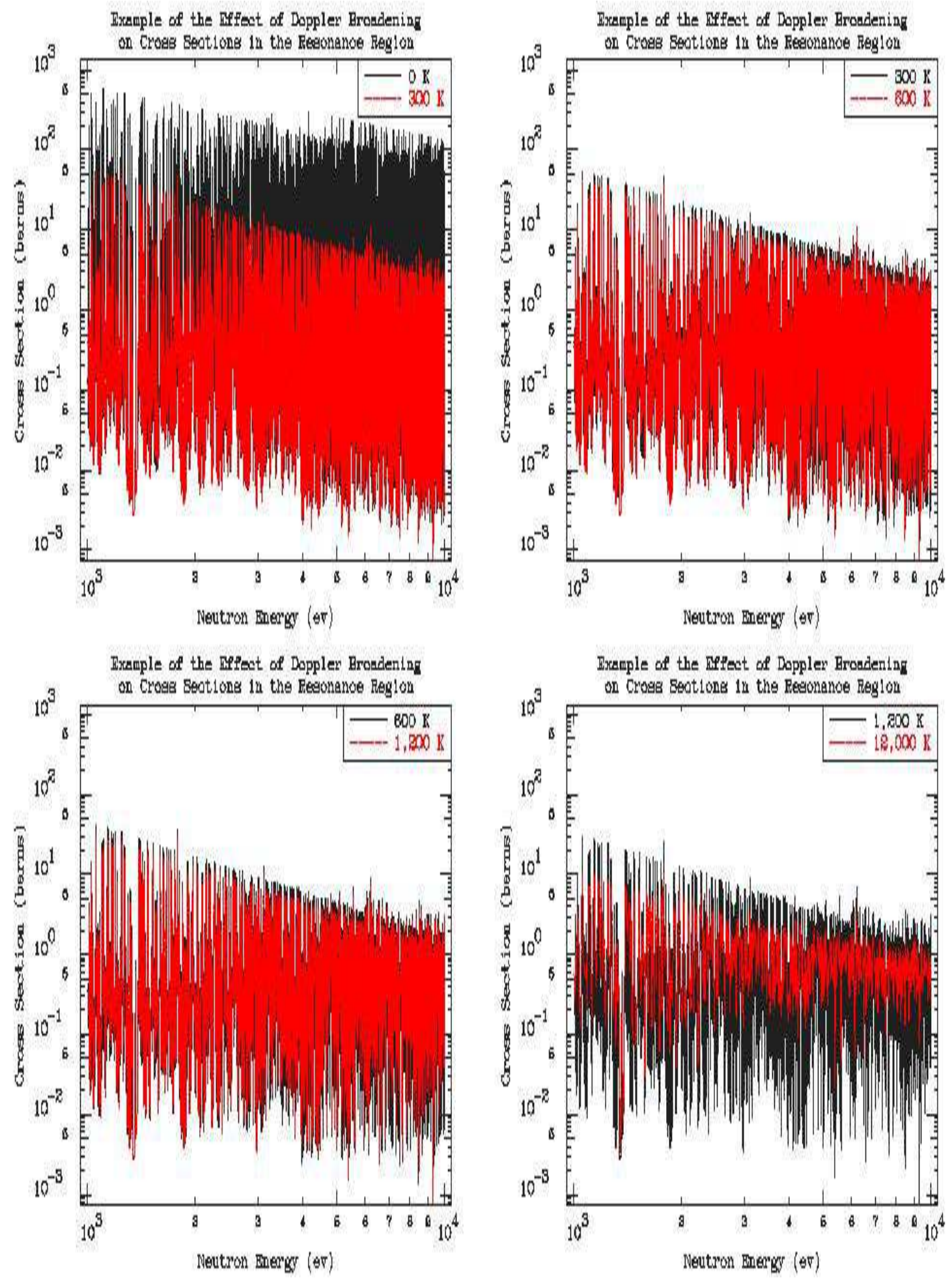
Fig.2: Effect of Doppler Broadening on Resonance Cross Sections
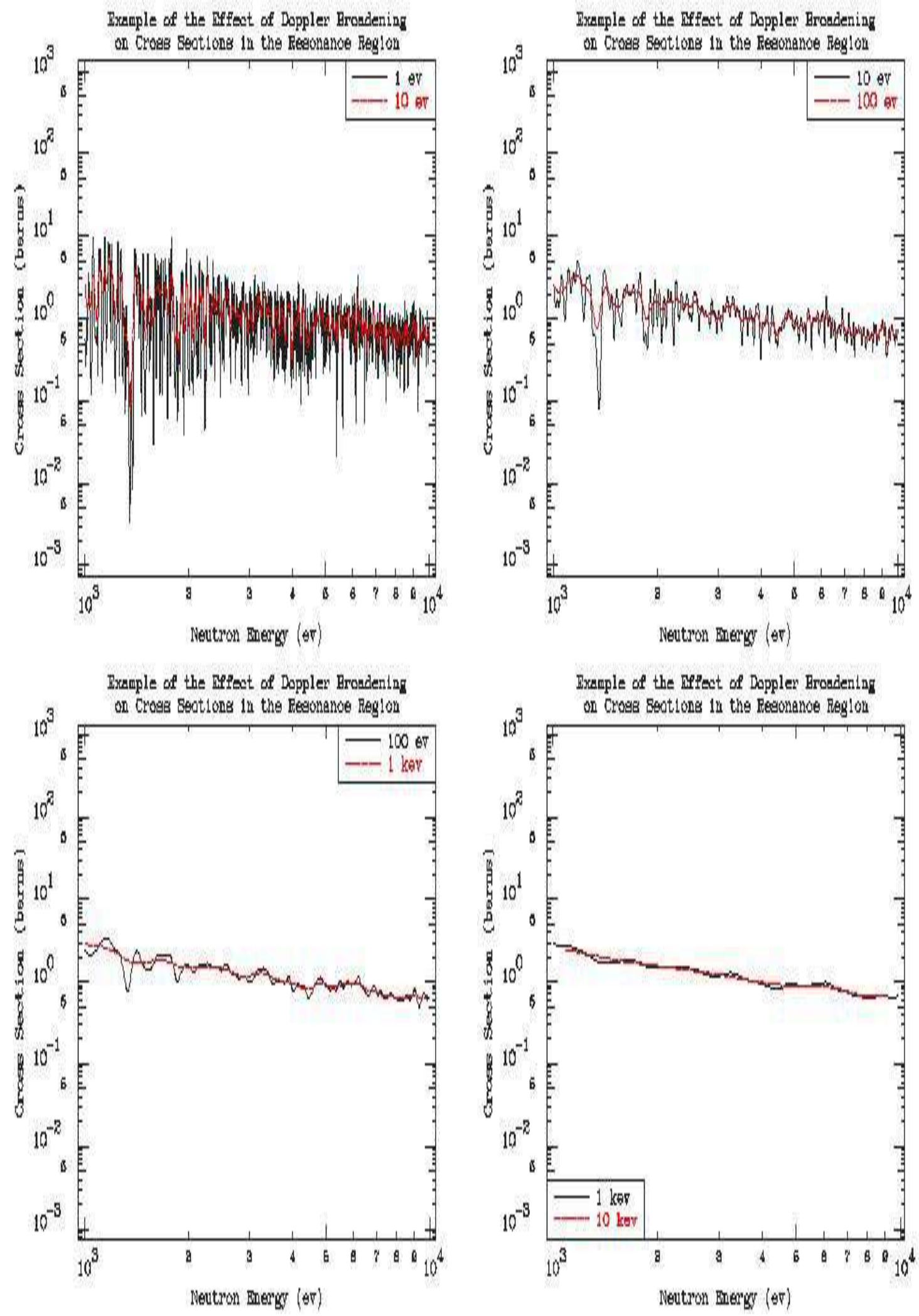
Fig.3: Effect of Doppler Broadening on Low Energy Cross Sections
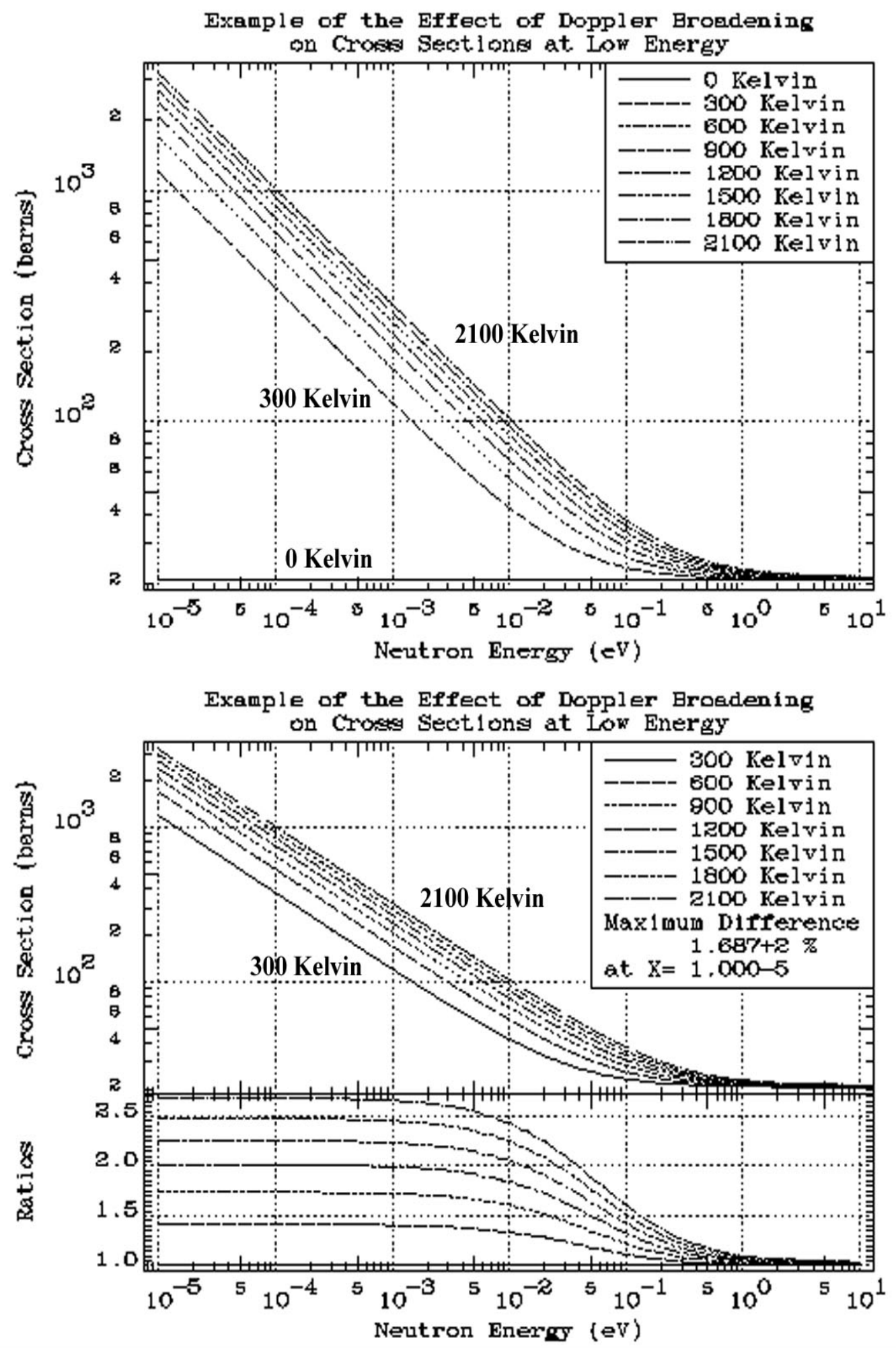
Fig.4: Effect of Doppler Broadening on Neutron Spectrum

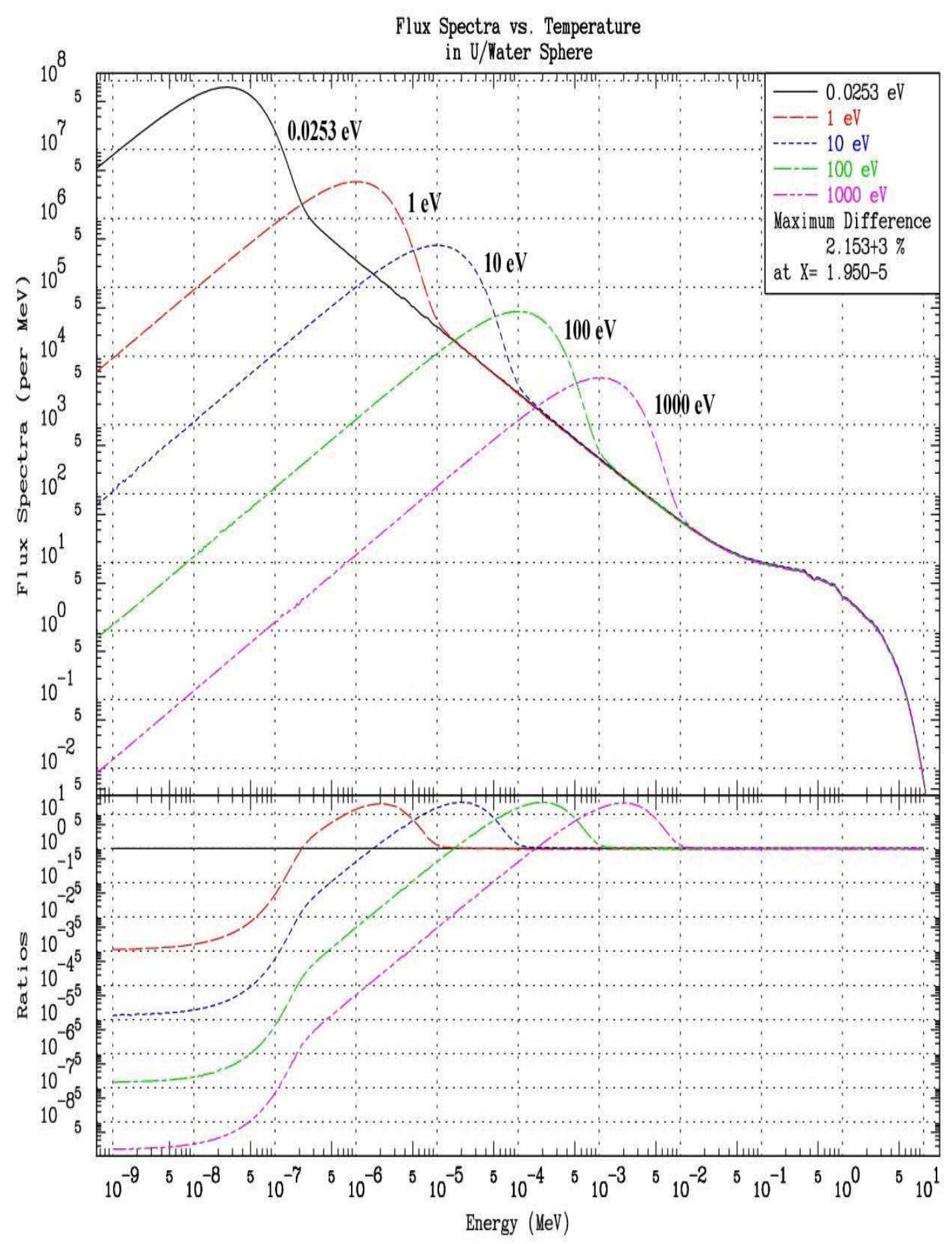

